\title{
Radiation Reaction, Renormalization and Poincaré Symmetry
}

Yurij YAREMKO

Institute for Condensed Matter Physics of National Academy of Sciences of Ukraine, 1 Svientsitskii Str., Lviv, 79011 Ukraine

E-mail: yar@ph.icmp.lviv.ua

Received July 08, 2005, in final form October 23, 2005; Published online November 01, 2005

Original article is available at http://www.emis.de/journals/SIGMA/2005/Paper012/

\begin{abstract}
We consider the self-action problem in classical electrodynamics of a massive point-like charge, as well as of a massless one. A consistent regularization procedure is proposed, which exploits the symmetry properties of the theory. The radiation reaction forces in both 4D and 6D are derived. It is demonstrated that the Poincaré-invariant six-dimensional electrodynamics of the massive charge is renormalizable theory. Unlike the massive case, the rates of radiated energy-momentum tend to infinity whenever the source is accelerated. The external electromagnetic fields, which do not change the velocity of the particle, admit only its presence within the interaction area. The effective equation of motion is the equation for eigenvalues and eigenvectors of the electromagnetic tensor. The interference part of energy-momentum radiated by two massive point charges arbitrarily moving in flat spacetime is evaluated. It is shown that the sum of work done by Lorentz forces of charges acting on one another exhausts the effect of combination of outgoing electromagnetic waves generated by the charges.
\end{abstract}

Key words: classical electrodynamics; point-like charges; Poincaré invariance; conservation laws; renormalization procedure

2000 Mathematics Subject Classification: 70S10; 78A40

\section{Introduction}

In classical electrodynamics particles interact with one another through the medium of a field, which has its own uncountable infinite degrees of freedom. The dynamics of entire system is governed by the action

$$
I=\sum_{a=1}^{2}\left(I_{a, \text { part }}+e_{a} \int \mathrm{d} \tau_{a} A_{\mu} \dot{z}_{a}^{\mu}\right)-\frac{1}{16 \pi} \int \mathrm{d}^{4} y f_{\mu \nu} f^{\mu \nu},
$$

which is invariant under ten infinitesimal transformations (space-time translations and rotations) that constitute the Poincaré group. These symmetry properties imply the conservation laws, i.e. those quantities that do not change with time. (In 4-dimensional Minkowski space $I_{a, \text { part }}$ is proportional to the worldline length, while in six dimensions the particle part of action (1) involves the term which is proportional to the curvature of particle's world line [1,2].)

Since the field $f_{a, \mu \nu}:=\partial_{\mu} A_{a, \nu}-\partial_{\nu} A_{a, \mu}$ generated by charge $e_{a}$ has a singularity on its world line, demanding that the total action (1) be stationary under a variation $\delta z_{a}^{\mu}\left(\tau_{a}\right)$ of the world line does not give sensible motion equations. To make sense of the retarded field's action on the particle, we should perform the regularization procedure. It can be accomplished via substitution of Liénard-Wiechert solutions for field variables in ten conserved quantities corresponding to 


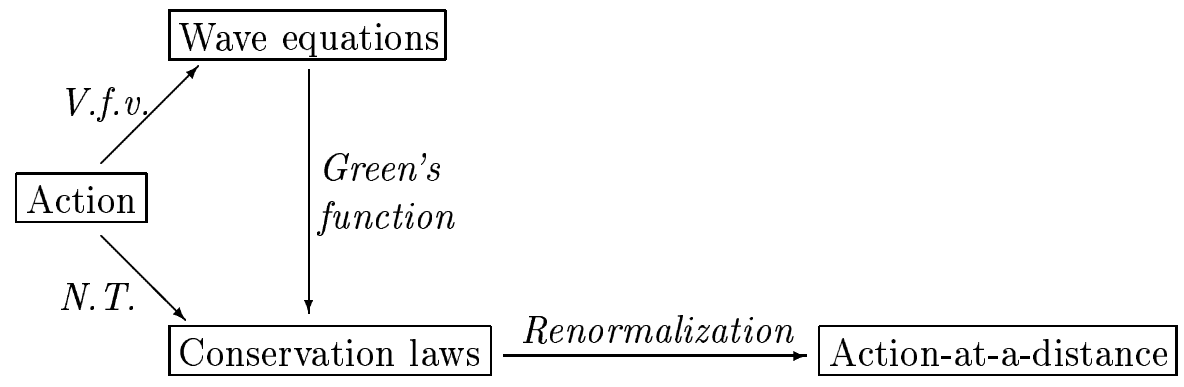

Figure 1. We start with standard action principle used in classical field theory. Variation with respect to field variables (V.f.v.) yields wave equations. The Poincaré invariance assures us, via Noether's theorem (N.T.), of conservation laws. The solutions of wave equations with point-like sources are substituted for field variables in these quantities, which do not change with time. Having performed renormalization procedure we derive the effective equations of motion which involve particles' characteristics only.

the symmetry of the action (1) under the Poincaré group (see Fig. 1). The conservation of the energy-momentum carried by the electromagnetic field [3]

$$
p_{\mathrm{em}}^{\nu}(\tau)=\int_{\Sigma} \mathrm{d} \sigma_{\mu} T^{\mu \nu}
$$

and the angular momentum tensor of the electromagnetic field [3]

$$
M_{\mathrm{em}}^{\mu \nu}(\tau)=\int_{\Sigma} \mathrm{d} \sigma_{\alpha}\left(x^{\mu} T^{\alpha \nu}-x^{\nu} T^{\alpha \mu}\right)
$$

corresponds to the symmetry of action (1) with respect to space-time translations and spacetime rotations, respectively. (By $\hat{T}$ we denote the electromagnetic field's stress-energy tensor; $\mathrm{d} \sigma_{\mu}$ is the outward-directed surface element on an arbitrary space-like hypersurface $\Sigma$.)

Noether quantity $G_{\mathrm{em}}^{\alpha}$ carried by electromagnetic field consists of terms of two quite different types [4]: (i) bound, $G_{\text {bnd }}^{\alpha}$, which are permanently "attached" to the sources and carried along with them; (ii) radiative, $G_{\text {rad }}^{\alpha}$, which detach themselves from the charges and lead independent existence. Within regularization procedure the bound terms are coupled with energy-momentum and angular momentum of "bare" sources, so that already renormalized characteristics $G_{\text {part }}^{\alpha}$ of charged particles are proclaimed to be finite. Noether quantities, which are properly conserved, become:

$$
G^{\alpha}=G_{\text {part }}^{\alpha}+G_{\text {rad }}^{\alpha}
$$

In the present paper we apply the regularization scheme based on the balance equations $\dot{G}^{\alpha}=0$ to the following problems:

- self-action in four- and six-dimensional classical electrodynamics;

- interference in the radiation of two point-like sources in 4-dimensional Minkowski space;

- radiation reaction for a photon-like charge in response to the external electromagnetic field.

\section{On the regularization procedure in classical electrodynamics}

We do not need assumptions concerning with the structure of singularity of the Maxwell energymomentum tensor density $\hat{T}$ on a particle trajectory $\zeta: \mathbb{R} \rightarrow \mathbb{M}_{4}$. Our consideration is founded on the energy-momentum and angular momentum balance equations for a closed system of 
accelerated charge $e$ coupled with electromagnetic field. They constitute the following system of differential equations $[5,6]$ :

$$
\begin{aligned}
& \dot{p}_{\text {part }}^{\mu}=-\frac{2}{3} e^{2} \ddot{z}^{2} \dot{z}^{\mu} \\
& \dot{z} \wedge p_{\text {part }}=-\frac{2}{3} e^{2} \dot{z} \wedge \ddot{z} .
\end{aligned}
$$

(Symbol $\wedge$ denotes the wedge product; we use an overdot on $z$ to indicate differentiation with respect to the evolution parameter $\tau$.) The solution of equation (3) involves an arbitrary scalar function, say $m$ :

$$
p_{\text {part }}^{\mu}=m(\tau) \dot{z}^{\mu}-\frac{2}{3} e^{2} \ddot{z}^{\mu} .
$$

This expression explains how already renormalized particle 4-momentum depends on worldline characteristics, i.e. on 4-velocity $\dot{z}:=u$ and 4-acceleration $\ddot{z}:=a$.

Since $\dot{z}^{2}=-1$, the scalar product of particle 4 -velocity on the first-order derivative of particle 4-momentum (2) is as follows:

$$
\left(\dot{p}_{\text {part }} \cdot \dot{z}\right)=\frac{2}{3} e^{2} \ddot{z}^{2}
$$

Since $(\dot{z} \cdot \ddot{z})=0$, the scalar product of particle acceleration on the particle 4 -momentum (4) is given by

$$
\left(p_{\text {part }} \cdot \ddot{z}\right)=-\frac{2}{3} e^{2} \ddot{z}^{2} .
$$

Summing up (5) and (6), we obtain

$$
\frac{\mathrm{d}\left(p_{\text {part }} \cdot \dot{z}\right)}{\mathrm{d} \tau}=0 \text {. }
$$

According to [7], the scalar product of the 4-momentum and the 4-velocity $\left(p_{\text {part }} \cdot \dot{z}\right)=m(\tau)$ is the rest mass of the particle. Since (7) the function $m$ is of constant value. Having substituted the expression (4) with $m=$ const into equation (2), we obtain the well-known Abraham 4-vector of radiation reaction [8].

\section{Interference in the radiation of two point-like sources}

The Maxwell energy-momentum tensor density $\hat{T}$ is quadratic in electromagnetic field strengths $\hat{f}=\hat{f}_{(1)}+\hat{f}_{(2)}$ where $\hat{f}_{(a)}$ denotes the retarded Liénard-Wiechert solution associated with the $a$-th particle. Hence $\hat{T}$ can be decomposed as follows [9]:

$$
T^{\mu \nu}=T_{(1)}^{\mu \nu}+T_{(2)}^{\mu \nu}+T_{\text {int }}^{\mu \nu} .
$$

By $\hat{T}_{(a)}$ we mean the contribution due to the field of the $a$-th particle [3]

$$
4 \pi T_{(a)}^{\mu \nu}=f_{(a)}^{\mu \lambda} f_{(a) \lambda}^{\nu}-\frac{1}{4} \eta^{\mu \nu} f_{(a)}^{\kappa \lambda} f_{\kappa \lambda}^{(a)}
$$

while interference term

$$
4 \pi T_{\mathrm{int}}^{\mu \nu}=f_{(1)}^{\mu \lambda} f_{(2) \lambda}^{\nu}+f_{(2)}^{\mu \lambda} f_{(1) \lambda}^{\nu}-\frac{1}{4} \eta^{\mu \nu}\left(f_{(1)}^{\kappa \lambda} f_{\kappa \lambda}^{(2)}+f_{(2)}^{\kappa \lambda} f_{\kappa \lambda}^{(1)}\right)
$$

describes the combination of outgoing electromagnetic waves. 
Aguirregabiria and Bel [9] prove the fundamental theorem that the "mixed" radiation rate does not depend on the shape of the space-like surface, which is used to integrate the Maxwell energy-momentum tensor density. To integrate the "mixed" radiation rate

$$
p_{\text {int }}^{\nu}=\int_{\Sigma} \mathrm{d} \sigma_{\mu} T_{\text {int }}^{\mu \nu}
$$

we use the hyperplane $\Sigma_{t}=\left\{y \in \mathbb{M}_{4}: y^{0}=t\right\}$ associated with an unmoving inertial observer. The "laboratory" time $t$ is a single common parameter defined along all the world lines $\zeta_{a}: \mathbb{R} \rightarrow$ $\mathbb{M}_{4}$ of the system. Since the delay in action ensures shifts in arguments in the electromagnetic field strengths, $a$-th world line $\zeta_{a}$ is parametrized by "individual" retarded time $t_{a}$.

The key to the problem is that the angle integration of the interference contribution (8) results the sum of partial derivatives in time variables:

$$
\int_{0}^{2 \pi} \mathrm{d} \varphi T_{\mathrm{int}}^{0 \nu}=\frac{\partial^{2} G_{0}^{\nu}}{\partial t_{1} \partial t_{2}}+\frac{\partial G_{1}^{\nu}}{\partial t_{1}}+\frac{\partial G_{2}^{\nu}}{\partial t_{2}}
$$

It allows us to calculate the interference part of energy-momentum carried by two-particle electromagnetic field. It consists of the bound terms and radiative terms [11]:

$$
p_{\text {int }}^{\nu}=p_{\text {int,bnd }}^{\nu}-\sum_{b \neq a} \int_{-\infty}^{t} \mathrm{~d} t_{a} F_{b a}^{\mu}
$$

The bound terms are absorbed by particles' 4-momenta within the renormalization procedure.

The radiative part of energy-momentum carried by "two-particle" field consists of the integrals of individual Larmor relativistic rates over corresponding world lines and the work done by Lorentz forces of point-like charges acting on one another [10, 11]:

$$
P^{\mu}=\sum_{a=1}^{2}\left[p_{a, \text { part }}^{\mu}(t)+\frac{2}{3} e_{a}^{2} \int_{-\infty}^{t} \mathrm{~d} t_{a}\left(a_{a} \cdot a_{a}\right) u_{a}^{\mu}\left(t_{a}\right)\right]-\sum_{b \neq a} \int_{-\infty}^{t} \mathrm{~d} t_{a} F_{b a}^{\mu} .
$$

Here $p_{a \text {,part }}$ denotes (already renormalized) four-momentum of the $a$-th charged particle. The integral of Larmor relativistic rate describes the contributions $\hat{T}_{(a)}$ due to the $a$-th individual field, while the sum of work done by Lorentz force due to the $b$-th particle acting on the $a$-th one expresses the joint contribution $\hat{T}_{\text {int }}$ due to combination of fields.

Having differentiated equation (9), we arrive at the relativistic generalization of Newton's second law

$$
\dot{p}_{a, \mathrm{part}}^{\mu}=-\frac{2}{3} e_{a}^{2}\left(a_{a} \cdot a_{a}\right) u_{a}^{\mu}+F_{b a}^{\mu},
$$

where loss of energy due to radiation is taken into account.

\section{Radiation reaction and renormalization in six-dimensional classical electrodynamics}

The Liénard-Wiechert potential in six dimensions depends on particle acceleration [1]; in mostly plus signature

$$
A_{\mu}=e\left[\frac{a_{\mu}(\tau)}{r^{2}}+\frac{u_{\mu}(\tau)}{r^{3}}\left(1+r a_{k}\right)\right] .
$$


Here $r$ is the retarded distance [3] and $a_{k}=a_{\alpha} k^{\alpha}$ is the component of the particle acceleration in the direction of $k^{\alpha}:=r^{-1}\left(y^{\alpha}-z^{\alpha}(\tau)\right)$.

We assume that an intrinsic structure of singularity of $\hat{T}$ in the immediate vicinity of particle's world line $\zeta: \mathbb{R} \rightarrow \mathbb{M}_{6}$ is beyond the limits of classical theory. The radiative part of electromagnetic field's energy-momentum is as follows $[12,13]$ :

$$
p_{\mathrm{rad}}^{\mu}=e^{2} \int_{-\infty}^{t} \mathrm{~d} \tau\left(\frac{4}{5}(\dot{a} \cdot \dot{a}) u^{\mu}-\frac{6}{35}(a \cdot a) \dot{a}^{\mu}+\frac{3}{7} a^{\mu}(a \cdot a)^{\cdot}+2(a \cdot a)^{2} u^{\mu}\right),
$$

where $(a \cdot a)^{\cdot}$ denotes the derivative of the square of particle acceleration with respect to evolution parameter $\tau$.

The radiative part of electromagnetic field's angular momentum depends on all previous motion of a source up to the instant of observation $t[12,13]$ :

$$
\begin{aligned}
M_{\mathrm{rad}}^{\mu \nu}= & e^{2}\left\{\int_{-\infty}^{t} \mathrm{~d} \tau\left(z^{\mu} P_{\mathrm{rad}}^{\nu}-z^{\nu} P_{\mathrm{rad}}^{\mu}\right)\right. \\
& \left.+\int_{-\infty}^{t} \mathrm{~d} \tau\left[\frac{4}{5}\left(a^{\mu} \dot{a}^{\nu}-a^{\nu} \dot{a}^{\mu}\right)+\frac{64}{35}(a \cdot a)\left(u^{\mu} a^{\nu}-u^{\nu} a^{\mu}\right)\right]\right\} .
\end{aligned}
$$

Here $P_{\text {rad }}$ denotes the integrand of equation (10).

With (11) in mind we assume that already renormalized angular momentum tensor of the particle has the form

$$
M_{\text {part }}^{\mu \nu}=z^{\mu} p_{\text {part }}^{\nu}-z^{\nu} p_{\text {part }}^{\mu}+u^{\mu} \pi_{\text {part }}^{\nu}-u^{\nu} \pi_{\text {part }}^{\mu} .
$$

In [2] the extra momentum $\pi_{\text {part }}$ is due to additional degrees of freedom associated with acceleration involved in Lagrangian function for rigid particle.

Scrupulous analysis of consistency of the energy-momentum balance equation and angular momentum balance equation reveals that six-momentum of charged particle contains two (already renormalized) constants, $m$ and $\mu$ :

$$
p_{\text {part }}^{\beta}=m u^{\beta}+\mu\left(-\dot{a}^{\beta}+\frac{3}{2}(a \cdot a) u^{\beta}\right)+e^{2}\left[\frac{4}{5} \ddot{a}^{\beta}-\frac{8}{5} u^{\beta}(a \cdot a)^{\cdot}-\frac{64}{35}(a \cdot a) a^{\beta}\right] .
$$

Having substituted the right-hand side of (12) for the particle's six-momentum in the energymomentum balance equation $\dot{p}_{\text {part }}+\dot{p}_{\text {rad }}=0$, we derive the Lorentz-Dirac equation of motion of a charged particle under the influence of its own electromagnetic field. An external device adds covariant external force $F_{\text {ext }}$ to the right-hand side of this expression [1]:

$$
\dot{p}_{\text {part }}^{\mu}+e^{2}\left(\frac{4}{5} u^{\mu} \dot{a}^{2}-\frac{6}{35} a^{2} \dot{a}^{\mu}+\frac{3}{7} a^{\mu}\left(a^{2}\right)^{\cdot}+2 a^{4} u^{\mu}\right)=F_{\text {ext }}^{\mu} .
$$

\section{Radiation reaction and renormalization for a photon-like charged particle}

Our consideration is based on the Maxwell equations with point-like source [14, equation (14)]

$$
\square A_{\mu}(x)=-4 \pi j_{\mu}(x),
$$

which governs the propagation of the electromagnetic field produced by a photon-like charge. Current density is zero everywhere, except at the particle's position where it is infinite

$$
j_{\mu}(x)=q \int \mathrm{d} \tau u_{\mu}(\tau) \delta[x-z(\tau)]
$$


and $\square:=\eta^{\alpha \beta} \partial_{\alpha} \partial_{\beta}$ is the wave operator. Unlike the massive case, the photon-like charge, say $q$, generates the far electromagnetic field

$$
\hat{f}=q \frac{a \wedge k+a_{k} u \wedge k}{r},
$$

which does not yield to divergent Coulomb-like self-energy. Hence the photon-like charge does not possess an electromagnetic "cloud" permanently attached to it. The renormalization procedure is not necessary because the photon-like source is not "dressed".

As a consequence, the Brink-Di Vecchia-Howe action term [15]:

$$
I_{\text {part }}=\frac{1}{2} \int \mathrm{d} \tau e(\tau) \dot{z}^{2}
$$

is consistent with the field and the interaction terms (1). Variation of (14) with respect to Lagrange multiplier $e(\tau) \neq 0$ yields the isotropy condition $\dot{z}^{2}=0$. The particle part (14) of the total action (1) describes already renormalized massless charge.

The retarded distance involved in equation (13)

$$
r=-\eta_{\alpha \beta}\left[x^{\alpha}-z^{\alpha}(s)\right] u^{\beta}(s)
$$

contains particle's characteristics (position, $z^{\alpha}$, and 4-velocity, $u^{\beta}$ ) taken at the retarded time, $s$, being the (causal) root of algebraic equation

$$
\eta_{\alpha \beta}\left[x^{\alpha}-z^{\alpha}(s)\right]\left[x^{\beta}-z^{\beta}(s)\right]=0 .
$$

Because of the isotropy condition, the retarded distance (15) vanishes on the ray in the direction of particle's 4-velocity taken at the instant of emission $z(s) \in \gamma$. Unlike the massive case, the field (13) diverges at all the points of this ray with vertex at the point of emission.

Volume integration of the radiative energy-momentum tensor density

$$
4 \pi T^{\alpha \beta}=\frac{q^{2}}{r^{2}} a^{2} k^{\alpha} k^{\beta}
$$

over a hyperplane $\Sigma_{t}=\left\{x \in \mathbb{M}_{4}: x^{0}=t\right\}$ gives the amount of radiated energy-momentum at fixed instant $t$. We introduce the set of curvilinear coordinates for flat space-time $\mathbb{M}_{4}$ involving the observation time $t$ and the retarded time $s$ :

$$
x^{\alpha}=z^{\alpha}(s)+(t-s) \Omega_{\alpha^{\prime}}^{\alpha} n^{\alpha^{\prime}} .
$$

The null vector $n:=(1, \boldsymbol{n})$ has the components $(1, \cos \varphi \sin \vartheta, \sin \varphi \sin \vartheta, \cos \vartheta) ; \vartheta$ and $\varphi$ are two polar angles which distinguish the points on the spherical wave front

$$
S(z(s), t-s)=\left\{x \in \mathbb{M}_{4}:\left(x^{0}-s\right)^{2}=\sum_{i}\left(x^{i}-z^{i}(s)\right)^{2}, x^{0}=t\right\},
$$

which is the intersection of the future light cones with vertex at point $z(s) \in \gamma$ and hyperplane $\Sigma_{t}$. Matrix space-time components are $\Omega_{0 \mu}=\Omega_{\mu 0}=\delta_{\mu 0}$; its space components $\Omega_{i j}$ constitute the orthogonal matrix which rotates space axes of the laboratory Lorentz frame until new $z$ axis is directed along three-vector $\boldsymbol{v}$. (Particle's 4-velocity has the form $\left(1, v^{i}\right),|\boldsymbol{v}|=1$, if parameterization of the world line $\gamma$ is provided by a disjoint union of hyperplanes $\Sigma_{t}$.) In terms of curvilinear coordinates $(t, s, \vartheta, \varphi)$ the retarded distance (15) is as follows:

$$
r=(t-s)(1-\cos \vartheta)
$$




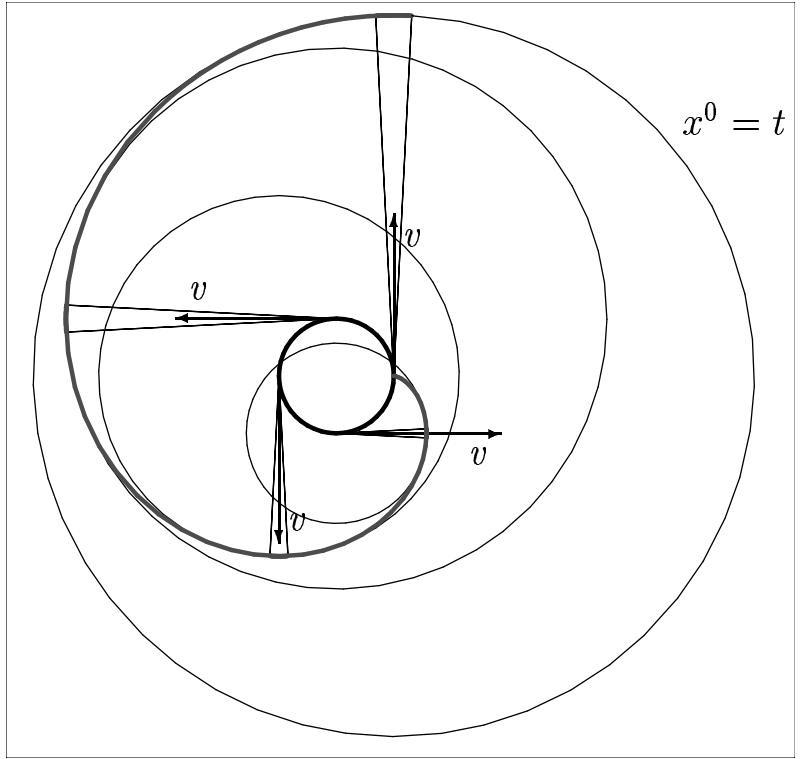

Figure 2. The bold circle pictures the trajectory of a photon-like charge. The others are spherical wave fronts viewed in the observation hyperplane $\Sigma_{t}=\left\{x \in \mathbb{M}_{4}: x^{0}=t\right\}$. The circling photon-like charge radiates infinite rates of energy-momentum and angular momentum in the direction of its velocity $\boldsymbol{v}$ at the instant of emission. The energy-momentum carried by electromagnetic field of accelerated charge tends to infinity on the spiral curve.

Angle integration reveals that the Larmor term tends to infinity whenever the photon-like charge is accelerated:

$$
\begin{aligned}
& p_{\mathrm{em}}^{0}=\frac{q^{2}}{2}\left(-\frac{1}{8}+\lim _{\vartheta \rightarrow 0} \frac{1}{2(1-\cos \vartheta)^{2}}\right) \int_{-\infty}^{t} \mathrm{~d} \tau a^{2}(\tau), \\
& p_{\mathrm{em}}^{i}=\frac{q^{2}}{2}\left(\frac{3}{8}-\lim _{\vartheta \rightarrow 0}\left[\frac{1}{1-\cos \vartheta}-\frac{1}{2(1-\cos \vartheta)^{2}}\right]\right) \int_{-\infty}^{t} \mathrm{~d} \tau a^{2}(\tau) v^{i}(\tau) .
\end{aligned}
$$

The situation is pictured in Fig. 2. Since the emitted radiation detaches itself from the charge and leads an independent existence, it cannot be absorbed within a renormalization procedure.

To change the velocity of the massless charge the energy is necessary which is too large to be observed, while non-accelerated photon-like charge does not generate the electromagnetic field. The evolution of the particle beyond an interaction area is determined by the BrinkDi Vecchia-Howe Lagrangian (14). The particle's 4-momentum $p_{\text {part }}^{\mu}=e(\tau) \dot{z}^{\mu}$ does not change with time: $\dot{e}(\tau) \dot{z}^{\mu}=0$. Since $\dot{z}^{\mu} \neq 0$, the Lagrange multiplier $e$ does not depend on $\tau$. We deal with a photon-like particle moving in the $\dot{z}$-direction with frequency $\omega_{0}=e_{0} \dot{z}^{0}$, such that its energy-momentum 4-vector can be written $p_{\text {part }}^{\mu}=\left(\omega_{0}, \omega_{0} \boldsymbol{k}\right),|\boldsymbol{k}|=1$.

When considering the system under the influence of an external device, the change in particle's 4-momentum is balanced by the external force:

$$
\dot{e} v^{\mu}=q F^{\mu}{ }_{\nu} v^{\nu} .
$$

This effective equation of motion is supplemented with the condition of absence of radiative damping. In other words, the external device admits a massless charge if and only if the components of null vector of 4-velocity do not change with time despite the influence of the external field.

The expression (16) is obtained in [16] where the model of magnetosphere of a rapidly rotating neutron star (pulsar) is elaborated. (The gas of ultrarelativistic electrons and positrons moving in a very strong electromagnetic field of the pulsar is meant.) If the gradient of star's potential 
is much larger than the particle's rest energy $m_{e} c^{2}$, the strong radiation damping suppresses the particle gyration. It is shown [16] that the particle velocity is directed along one of the eigenvectors of (external) electromagnetic tensor $\hat{F}$ if $m_{e} \rightarrow 0$ in the Lorentz-Dirac equation. Equation (16) on eigenvalues and eigenvectors of the electromagnetic tensor governs the motion of charges in zero-mass approximation. This conclusion is in contradiction with that of Ref. [14], where the radiation back reaction is finite and the 5 -th order differential equation determines the evolution of photon-like charge. The reason is that the Dirac regularization approach to the radiation back reaction (one-half retarded field minus one-half advanced one), employed by Kazinski and Sharapov, is not valid in the case of the photon-like charged particle and its field. Indeed, the advanced field is free from the ray singularity, and the Dirac combination does not yield a finite part of the self force.

\subsection{Conformal invariance}

Action integral (1) with $I_{\text {part }}$ in form of (14) is conformally invariant. According to [17], conformal group $\mathcal{C}(1,3)$ consists of Poincaré transformations (time and space translations, space and mixed space-time rotations), dilatations

$$
x^{\prime \mu}=e^{\theta} x^{\mu}
$$

and conformal transformations

$$
x^{\prime \mu}=\frac{x^{\mu}-b^{\mu}(x \cdot x)}{D}, \quad D=1-2(x \cdot b)+(x \cdot x)(b \cdot b) .
$$

(The scalar $\theta$ and 4 -vector $b$ are group parameters.)

The components of electromagnetic field are transformed as follows:

$$
F_{\alpha \beta}=e^{2 \theta} F_{\alpha \beta}^{\prime}, \quad F_{\alpha \beta}=F_{\mu \nu}^{\prime} \Omega_{\alpha}^{\mu} \Omega_{\beta}^{\nu}
$$

where matrix

$$
{\Omega^{\mu}}_{\alpha}:=\frac{\partial x^{\prime \mu}}{\partial x^{\alpha}}=D^{-1} \lambda^{\mu}{ }_{\beta}\left(x^{\prime \prime}\right) \lambda^{\beta}{ }_{\alpha}(x), \quad x^{\prime \prime}=\frac{x}{(x \cdot x)}-b, \quad \lambda_{\alpha}^{\beta}(x)=\delta^{\beta}{ }_{\alpha}-\frac{2 x^{\beta} x_{\alpha}}{(x \cdot x)}
$$

satisfies the condition

$$
\eta_{\mu \nu} \Omega^{\mu}{ }_{\alpha} \Omega_{\beta}^{\nu}=D^{-2} \eta_{\alpha \beta}
$$

Since

$$
\dot{z}^{\prime \mu}=e^{\theta} \dot{z}^{\mu} \quad \dot{z}^{\prime \mu}=\Omega^{\mu}{ }_{\alpha} \dot{z}^{\alpha}
$$

the Lagrange multiplier $e(\tau)$ involved in the Brink-Di Vecchia-Howe action term (14) transforms as

$$
e(\tau)=e^{2 \theta} e^{\prime}(\tau), \quad e(\tau)=D^{-2} e^{\prime}(\tau)
$$

Direct calculation shows that the effective equation of motion (16) is invariant with respect to dilatation (17) and conformal transformation (18).

It is worth noting that the conformal invariance yields conservation laws, which are functions of energy-momentum and angular momentum conserved quantities.

Conformal invariance of our particle plus field system reinforces our conviction that the backreaction force vanishes. Indeed, the appropriate renormalization procedure should preserve this symmetry property while the the Brink-Di Vecchia-Howe action term (14) does not contain a parameter to be renormalized. Therefore, the photon-like charge must not radiate. 


\section{Conclusions}

The law of conservation of the total four-momentum of a composite (particle plus field) system provides the foundation for Dirac's derivation [8] of the radiation-reaction force. It involves the Taylor expansion of a finite sized charged sphere, in which the first two terms lead to the electromagnetic self-energy, and the Abraham radiation reaction four-vector, respectively. Inevitable infinities arising in six-dimensional electrodynamics are stronger than in four dimensions. In [1] Kosyakov calculates the flux of energy-momentum and derives the radiation-reaction force in six-dimensions by adding of appropriate Schott term.

In the present paper we consider also the conserved quantities corresponding to the invariance of the theory under proper homogeneous Lorentz transformations. They gives additional information which allows us to avoid the problems concerning with an intrinsic structure of a singularity. In such a way we reformulate the problem of renormalizability within the problem of Poincaré invariance of a closed particle plus field system. The conservation laws are an immovable fulcrum about which tips the balance of truth regarding renormalization and radiation reaction.

The renormalization scheme is applied to the problem of combination of outgoing electromagnetic waves generated by two point-like sources $[10,11]$. The evaluation of the interference part of energy-momentum radiated by charges is not a trivial matter, since delay in action ensures shifts in arguments in the electromagnetic field strengths. Rigorous calculations show that the interference rate of radiation which escapes to infinity is equal to the rate of work done by Lorentz forces of charges acting on one another. The bound terms arise, which can be interpreted as a usual deformation of the bound electromagnetic "clouds" of charges due to mutual interaction. They are absorbed within the renormalization procedure, as well as the inevitable infinities arising in a one-particle problem.

Inspection of the energy-momentum and angular momentum carried by the electromagnetic field of a photon-like charge reveals the reason why it is not yet detected (if it exists). Noninteracting massless charges do manifest themselves in no way. Any external electromagnetic field (including that generated by a detecting device) will attempt to change the velocity of the charged particle. Whenever the effort will be successful, the radiation reaction will increase extremely. In general, this circumstance forbids the presence of the photon-like charges within the interaction area.

To survive, photon-like charges need an extremely strong field of specific configuration, as that of the rotating neutron star (pulsar). In [16] the model of the pulsar magnetosphere is elaborated. It involves the so-called dynamical phase, which consists of the massless charged particles moving with speed of light along real eigenvalues of the electromagnetic field tensor of the star.

\section{Acknowledgements}

The author would like to thank Professors B.P. Kosyakov, V. Tretyak, Dr. A. Duviryak, and Professor Yu.A. Rylov for helpful discussions and critical comments.

[1] Kosyakov B.P., Exact solutions of classical electrodynamics and the Yang-Mills-Wong theory in evendimensional spacetime, Teor. Mat. Fiz., 1999, V.119, N 1, 119-135 (English transl.: Theor. Math. Phys., 1999, V.119, N 1, 493-509); for revised version see hep-th/0207217.

[2] Nesterenko V.V., Curvature and torsion of the world curve in the action of the relativistic particle, J. Math. Phys., 1991, V.32, N 12, 3315-3320.

[3] Rohrlich F., Classical charged particles, Redwood City, Addison-Wesley, 1990.

[4] Teitelboim C., Splitting of the Maxwell tensor: radiation reaction without advanced fields, Phys. Rev. D, 1970, V.1, N 6, 1572-1582. 
[5] Yaremko Yu., On the validity of the Lorentz-Dirac equation, J. Phys. A: Math. Gen., 2002, V.35, 831-839; Corrigendum, J. Phys. A: Math. Gen., 2003, V.36, 5159.

[6] Yaremko Yu., On the regularization procedure in classical electrodynamics, J. Phys. A: Math. Gen., 2003, V.36, 5149-5156.

[7] Kosyakov B.P., On the inert properties of particles in classical theory, 2003, Fiz. Elem. Chast. $i$ Atom. Yadra, V.34, 1564 (English transl.: Phys. Part. Nucl., 2003, V.34, 808); hep-th/0208035.

[8] Dirac P.A.M., Classical theory of radiating electrons, Proc. R. Soc. A, 1938, V.167, 148-169.

[9] Aguirregabiria J.M., Bel L., Electromagnetic energy and linear momentum radiated by two point charges, Phys. Rev. D, 1984, V.29, N 6, 1099-1106.

[10] Yaremko Yu., Interference in the radiation of two point-like sources, J. Phys. A: Math. Gen., 2004, V.37, L531-L538.

[11] Yaremko Yu., Interference of outgoing electromagnetic waves generated by two point-like sources, Int. J. Mod. Phys. A, 2005, V.20, N 1, 129-159.

[12] Yaremko Yu., Radiation reaction, renormalization and conservation laws in six-dimensional classical electrodynamics, J. Phys. A: Math. Gen., 2004, V.37, 1079-1091.

[13] Yaremko Yu., Radiation reaction and renormalization via conservation laws of the Poincaré group, J. Phys. Stud., 2004, V.8, N 3, 203-210.

[14] Kazinski P.O., Sharapov A.A., Radiation reaction for a massless charged particle, Class. Quantum Grav., 2003, V.20, 2715-2725.

[15] Brink L., Deser S., Zumino B., Di Vecchia P., Howe P., Local supersymmetry for spinning particles, Phys. Lett. B, 1976, V.64, N 4, 435-438.

[16] Rylov Yu.A., The algebraical structure of the electromagnetic tensor and description of charged particles moving in the strong electromagnetic field, J. Math. Phys., 1989, V.30, N 2, 521-536.

[17] Fushchich W.I., Nikitin A.G., Symmetry of equations of quantum mechanics, Moscow, Nauka, 1990 (in Russian). 